\title{
Functionalization of Mesoporous Si-MCM-41 by Grafting with Trimethylchlorosilane
}

\author{
Nurul Izza Taib (Corresponding author) \\ Faculty of Applied Sciences, Universiti Teknologi MARA, 40450 Shah Alam \\ Selangor Darul Ehsan, Malaysia \\ Tel: 603-5543-5765_E-mail: izza257@salam.uitm.edu.my \\ Salasiah Endud \\ Department of Chemistry, Faculty of Science, Universiti Teknologi Malaysia \\ 81310 UTM Skudai, Johor, Malaysia \\ Tel: 607-553-4503 E-mail: salasiah@kimia.fs.uitm.my \\ Md. Nasir Katun \\ Department of Chemistry, Faculty of Science, Universiti Teknologi Malaysia \\ 81310 UTM Skudai, Johor, Malaysia \\ Tel: 607-553-4502Ｅ-mail: nasir@kimia.fs.uitm.my
}

Received: January 18, 2011 Accepted: February 10, 2011 doi:10.5539/ijc.v3n3p2

\begin{abstract}
The production of functionalized mesoporous silicas has attracted great attention in the last years. In particular, the uses of organosilanes, as modification agents, has been largely studied due mainly to the increasing requirements deriving from the area of polymer nanocomposites, treatments of heavy metal contaminants, heterogeneous catalysis and also in components of active corrosion protection coatings. In this study, organosilane-modified mesoporous were prepared and investigated by various physico-chemical techniques, including X-ray powder diffractometry (XRD), Fourier transform infrared spectroscopy (FTIR), ${ }^{13} \mathrm{C}$ CP/MAS NMR and Thermalgravimetry (TGA). The organically modified mesoporous derived from the reaction of mesoporous Si-MCM-41 with trimethylchlorosilane have been considered. The results indicated that the surface modification did not modify the original structure of Si-MCM-41 but altered their original hydrophilic nature to hydrophobic. Herein, we demonstrate the possibility of modify and functionalize surface of MCM-41, enabling applications in many fields as nanocomposites, drug releasing agents and components of active coatings.
\end{abstract}

Keywords: MCM-41, Trimethylchlorosilane, Functionalized

\section{Introduction}

The preparation of mesoporous materials family M41S since 1992 (Kresge, et al., 1992) until now has opened a wide field of applications (Lazaridis, et al., 2009; Zhao, et al., 1996; Corma, 1997). Mesoporous molecular sieves have relatively large and uniform pore size $(15-100 \AA)$, which break past the pore size constraint $(<15 \AA)$ of microporous zeolites, high surface areas $\left(>1000 \mathrm{~m}^{2} \cdot \mathrm{g}^{-1}\right)$, highly ordered pore structures and very narrow pore size distributions (Juang, et al., 2006). Such materials have been prepared using cationic surfactant species to produce mesoscopically ordered lamellar (MCM-50), hexagonal (MCM-41), or cubic (MCM-48) structures in approximately micrometer-sized domains (Hoffmann, et al., 2006).

As one of these materials, MCM-41 is characterized by high pore volume, moderate hydrophobic character as well as parallel and ideally shaped pore structures without the complications of a network. Mesoporous silica can be synthesized via polycondensation of silica species which originate from different sources of silica such as sodium silicate, fumed silica or tetraethylorthosilicate (TEOS) and Ludox in the presence of surfactants as 
structure-directing agents. Many types of ionic surfactant such as hexadecyltrimetylammonium bromide and non-ionic surfactants such as amphiphilic triblock copolymers have been used for obtaining mesoporous silica with different pore structure and morphological characteristics.

Mesoporous silicas have been functionalized with both inorganic and organic species in order to provide additional physical or chemical properties to the silica parent material. These new hybrid organic-inorganic mesoporous ordered structures have attracted increasing attention because of their high surface area and the functionalized pore channels of large diameter. The high surface area allows the binding of a large number of surface groups and the functionalized pore channels of large diameter allow an easy reaction with adsorbates. The grafting of the functional groups to the pore walls of the mesoporous adsorbents can be fulfilled by the reaction between the hydrolyzable moieties and the surface silanol groups of the mesostructures.

In general, functionalization of these materials can be carried out by two independent methods (Lim \& Stein, 1999). The first of them is carried out by grafting the surface of the preformed silica by means of silanol groups reactions with an organoalkoxysilane compound supporting the active functional group. The second method consists in the simultaneous co-condensation within the synthesis medium of the alkoxysilane precursor of the silica mesostructure and a selected organoalkoxysilane to obtain the functionalized material after one step.

In this study, mesoporous pure silica Si-MCM-41 was chemically modified by functionalization with trimethylchlorosilane (TMCS). The synthesized material were investigated by various physico-chemical techniques, including X-ray powder diffractometry (XRD), Fourier transform infrared spectroscopy (FTIR), ${ }^{13} \mathrm{C}$ CP/MAS NMR and Thermalgravimetry (TGA). The results indicated that the surface modification did not modify the original structure of Si-MCM-41 but altered their original hydrophilic nature to hydrophobic.

\section{Experimental}

\subsection{Preparation of mesoporous pure silica Si-MCM-41}

The parent Si-MCM-41 was synthesized according to the following procedures. First, sodium silicate in a clear solution was prepared by dissolving $30.0450 \mathrm{~g}$ of Ludox $\left(30 \mathrm{wt} \% \mathrm{SiO}_{2}\right)$ in $3.0000 \mathrm{~g}$ of aqueous sodium hydroxide $1 \mathrm{M}$ solution (pellet from Merck) and heated at $80{ }^{\circ} \mathrm{C}$ for two hours. The surfactant solution was prepared by dissolving $9.1115 \mathrm{~g}$ of cetyltrimethylammonium bromide (CTABr) purchased from BDH Analar and $1.0500 \mathrm{~g}$ of $25 \mathrm{wt} \%$ aqueous $\mathrm{NH}_{3}$ solution (Merck) in a polypropylene bottle and the mixture was then heated with stirring for one hour at $80{ }^{\circ} \mathrm{C}$. After cooling to room temperature, sodium silicate was added dropwise to a polypropylene bottle containing surfactant solution. The tightly closed bottle was stirred vigorously at room temperature before heating in oven at $97{ }^{\circ} \mathrm{C}$ for one hour in static condition. $\mathrm{pH}$ adjustment to 10.2 by dropwise addition of acetic acid $25 \mathrm{wt} \%\left(\mathrm{CH}_{3} \mathrm{COOH}\right)$ (Merck) was performed and resulting gel mixture was heated for two days. Gel mixture had a molar composition of $6 \mathrm{SiO}_{2}: 1 \mathrm{CTABr}: 1.5 \mathrm{Na}_{2} \mathrm{O}: 0.15\left(\mathrm{NH}_{4}\right)_{2} \mathrm{O}: 250 \mathrm{H}_{2} \mathrm{O}$. After two days, siliceous MCM-41 was filtered, washed with distilled water until it neutralized and dried in an oven at $97{ }^{\circ} \mathrm{C}$ for 16 hours. The template was removed through calcination in air under static conditions at $550{ }^{\circ} \mathrm{C}$ for six hours before further application.

\subsection{Modification of Si-MCM-41}

A mixture of $1.5 \mathrm{~g}$ of as-synthesized MCM- 41 and $54 \mathrm{~mL}$ of trimethylchlorosilane (TMCS) was refluxed at $45{ }^{\circ} \mathrm{C}$ for overnight. $27 \mathrm{~mL}$ of pyridine was added and refluxed for another 18 hour. The resulting solid was then filtered, washed with hexane and absolute ethanol and dried.

\subsection{Characterization}

The solid samples were identified by powder XRD using a Bruker D8 Advance Diffractometer with $\mathrm{Cu}-\mathrm{K}_{\alpha}$ radiation $(\lambda=0.15418 \mathrm{~nm})$ at $40 \mathrm{kV}$ and $40 \mathrm{~mA}$. The FTIR spectra of the solid samples prepared by $\mathrm{KBr}$ technique were recorded on Shidmadzu Fourier Transform Infrared spectrometer. In this technique a pinch of sample was mixed with $\mathrm{KBr}$ salt and pelletized and IR spectra of the pellet were taken. Thermal stability of the samples was measured by using Pyris Diamond TG/DTA-Perkin Elmer under nitrogen flow $(35 \mathrm{~mL} / \mathrm{min})$ with heating rate $20{ }^{\circ} \mathrm{C} / \mathrm{min}$ from room temperature to $900{ }^{\circ} \mathrm{C}$. Solid State ${ }^{13} \mathrm{C}$ CP/MAS NMR experiments were performed using Bruker Advance $400 \mathrm{MHz} 9.4 \mathrm{~T}$ spectrometer. The ${ }^{13} \mathrm{C} \mathrm{CP} / \mathrm{MAS}$ NMR spectra were recorded with a recycle delay of $5.0 \mathrm{~s}$, number of transient of 6000 and spinning rate of $7 \mathrm{kHz}$. Chemical shifts for ${ }^{13} \mathrm{C}$ was referred to TMS. Thermo Finnigan - Surface Area Analyzer instrument was used for the single point BET surface area analysis. It's been done at $77 \mathrm{~K}$ (boiling temperature of liquid nitrogen) to determine surface area. Prior to the determination of single point BET surface area, the sample was out gassed at $200{ }^{\circ} \mathrm{C}$ for 20 minutes. 


\section{Results and discussion}

\subsection{Powder X-ray Diffraction (XRD)}

The X-ray Diffraction patterns obtained for siliceous MCM-41 before and after calcination in air at $550{ }^{\circ} \mathrm{C}$ are given in Figure 1. The diffractogram in Figure 1(a) shows the presence of a single high intensity and three weak peaks. In the low 2-theta region of $1.5^{\circ}-10^{\circ}$, the XRD pattern exhibits four diffraction peaks which could be indexed as (100), (110), (200) and (210) reflections, respectively. This is charateristics of long range ordered hexagonal MCM-41 mesoporous phase. The parent MCM-41 exhibits a narrow and strong peak at $41.03 \AA$

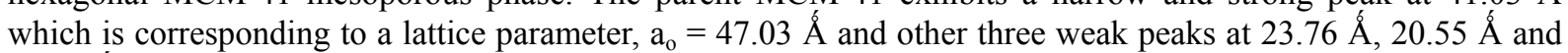
15.74 Á due to the (100), (110), (200) and (210) reflections, respectively.

The presence of same peaks in the siliceous MCM-41 after calcination with the increasing significantly the intensity of (100) peak and $d_{100}$ value shifted to the higher value shows that the removal of organic surfactant template and condensation of silanol groups in the pore walls. This can lead to the rearrangement of silica walls of siliceous MCM-41 thus increased the crystallinity of MCM-41. Kleitz, et al. reported that an initial change occurred up to $250{ }^{\circ} \mathrm{C}$ with the increase in intensity for all XRD reflections which indicate the increasing of crystallinity (Kleitz, et al., 2001). Calcination of Si-MCM-41 containing large amounts of carbonaceous species can leave carbon deposits or coke as contaminant in the pores and pore blocking may occur. When the surfactant template is removed by calcination, the low angle reflection intensities increase and the mesoporous ordered stucture could be dramatically affected (Kleitz, et al., 2003).

Figure 2 shows diffractogram of siliceous MCM-41 modified by silylation with trimethylchlorosilane (TMCS). The four main peaks still remained even after silylation process with increasing the intensity of (100) peak. This suggests that long range order of mesopores is still preserved and the silylation has no adverse effects on the ordered mesoporous structure of MCM-41. This shows also that TMCS only interacts on to the surface of calcined siliceous MCM-41. The XRD pattern is in excellent agreement with the parent MCM-41 which suggests that highly ordered MCM-41 can still be obtained after calcination and modification by silylation with TMCS.

\subsection{Fourier Transform Infrared (FTIR) Spectroscopy}

The FTIR spectra of siliceous MCM-41 before and after calcination and the modified product by silylation with trimethylchlorosilane (TMCS) are illustrated in Figure 3. For parent MCM-41, bands observed at $1234.3 \mathrm{~cm}^{-1}$ and $1071.4 \mathrm{~cm}^{-1}$ are characteristics peaks of asymmetric Si-O-Si stretching. Another characteristics peak is the symmetric Si-O-Si stretching observed at $785.9 \mathrm{~cm}^{-1}$. The peak at $456.1 \mathrm{~cm}^{-1}$ is characteristic of Si-O-Si bending. The broad band around $3500 \mathrm{~cm}^{-1}$ may be due to surface silanols and adsorbed water molecules which indicating the silica framework is hydrophillic.

For calcined siliceous MCM-41, the disappearance of peak at $2922.9 \mathrm{~cm}^{-1}$ and $2852.5 \mathrm{~cm}^{-1}$ can be concluded that the calcination at $550{ }^{\circ} \mathrm{C}$ is complete. This indicates that the organic template has been removed completely due to calcination. MCM-41 tends to adsorb water vapours in air since the surface of silica framework is water liking, the stretching mode of $\mathrm{H}_{2} \mathrm{O}$ is observed at $1635.5 \mathrm{~cm}^{-1}$. From the silylated spectrum, we can see the existence new peak around $2965.4 \mathrm{~cm}^{-1}$ characteristics of $\mathrm{CH}$ of methyl group. The stretching mode at $846.7 \mathrm{~cm}^{-1}$ and $758.9 \mathrm{~cm}^{-1}$ characteristics of Si-C stretching vibrations which are absent in the spectrum of siliceous MCM-41 before and after calcination. The presence of these new peaks indicates that there is interaction between TMCS and silanol group on wall surface of MCM-41. This suggest that the $-\mathrm{Si}\left(\mathrm{CH}_{3}\right)_{3}$ groups have successfully grafted onto the surface of MCM-41 which this can increases the hydrophobicity of Si-MCM-41 to facilitate the threading of any organic material into the channel of MCM-41.

\section{$3.3{ }^{13} \mathrm{C} C P / M A S$ Solid-state NMR}

From the spectrum in Figure 4, only single and sharp peak was observed at $-1.111 \mathrm{ppm}$ corresponds to carbon of methyl group in TMCS that anchored on the silica surface of Si-MCM-41 (Sutra, et al., 1999; Bu \& Rhee, 2000). The existence of one peak indicates that local environment of carbon of methyl group in TMCS is same. The

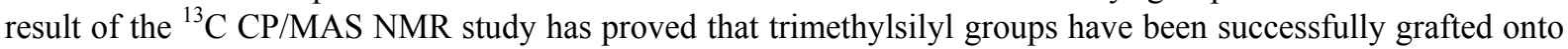
the Si-MCM-41. It also showed that the organic surfactant template has been removed completely due to calcination as there is no other peaks exist.

\subsection{Thermogravimetric Analysis (TGA)}

Figure 5 (a) shows the TGA profiles of the siliceous MCM-41 in nitrogen atmosphere showing three stages of weight loss. The first weight loss was observed at $50-150{ }^{\circ} \mathrm{C}$ about $3.1031 \%$ due to desorption of physisorbed water on the external surface of the crystallites or occluded in the macropores and mesopores present between 
the crystallites aggregates (Selvaraj, et al., 2003; Chu, et al., 2003). Meanwhile, in the range of 150-330 ${ }^{\circ} \mathrm{C}$ weight losses about $30.9060 \%$ are attributed to the decomposition or removal of organic (Guo, et al., 2001; He, et al., 2000). At the temperature range $330-550{ }^{\circ} \mathrm{C}$ is due to residual surfactant decomposition and water loss from the condensation of adjacent silanol group to form siloxane bond about $4.5197 \%$. The total weight loss up to $900{ }^{\circ} \mathrm{C}$ of this sample is in $39 \%$. There was almost no weight loss after $550{ }^{\circ} \mathrm{C}$ indicating that surfactant had been removed completely.

Figure 5 (b) shows the TGA profiles of calcined siliceous MCM-41. There is no significant difference in the curve pattern in the thermogram collected. Although water absorbed was detected in FTIR analysis was broader but thermogravimetric analysis shows little mass loss about $0.4231 \%$ after given thermal treatment. At higher temperatures (above $100{ }^{\circ} \mathrm{C}$ ), the mass loss $(0.6731 \%)$ can still be observed although the template have been removed. This could be due to the decomposition of coke but the loss was too small indicating that the sample was stable towards heat.

Figure 5 (c) shows the TGA profiles of silylated MCM-41 which shows weight loss under $100{ }^{\circ} \mathrm{C}$ about $0.9883 \%$ due to loss of physisorbed water molecules. The very gradual degradation rate observed at temperatures over the range between 100 to $550{ }^{\circ} \mathrm{C}$ corresponds to removal of the TMCS groups attached to Si-MCM-41 (Kang \& Choi, 2000). Meanwhile, further decomposition was observed after $550{ }^{\circ} \mathrm{C}$ due to the thermal dehydroxylation of internal surface silanol groups to form siloxane bridges about $4.0993 \%$. The adsorbed water on the external surface for the parent MCM- 41 was approximately more $3 \%$ and less than $1 \%$ after silylation treatment. This can confirm the hydrophobicity enhancement obtained by replacing surface silanol groups with trimethylsilyl groups (Jeong, et al., 2000).

\subsection{BET surface area}

The specific BET surface areas of as-synthesized calcined and silylated Si-MCM-41 are summarized in Table 1. The surface area for all the samples were determined by single-point BET method. The results show that the surface area of Si-MCM-41 was significantly increased after calcinations $\left(1083.54 \mathrm{~m}^{2} / \mathrm{g}\right)$. This may due to the removal of the organic template, $\mathrm{CTABr}$, which resulted in an increase in the adsorption site for the nitrogen molecules and hence the surface area of the calcined Si-MCM-41 sample (Shylesh \& Singh, 2004). The finding is well in agreement with the XRD, FTIR and TGA which also give indications of the removal of organic template from the material. On the other hand, the surface area of silylated Si-MCM-41 decreased to 975.42 $\mathrm{m}^{2} / \mathrm{g}$ with respect to that of the calcined sample as expected if the surface silanol groups were replaced by the larger trimethylsilyl groups. The silylation by trimethylchlorosilane may have induced the trimethylsilyl groups to occupy defect sites in the pore wall as self-assembled monolayers and decreased the adsorption site for the nitrogen molecules contributing to the decrease in surface area. The result also suggests that the silylation treatment has not altered the ordered structure of Si-MCM-41 as has been shown by the XRD pattern depicted in Figure 2.

\section{Conclusion}

Siliceous MCM-41 was successfully synthesized from Ludox $\left(30 \mathrm{wt} \% \mathrm{SiO}_{2}\right)$ as silica sources. Preparation and characterization of silylated Si-MCM-41 have been carried out. Silylated Si-MCM-41 was synthesized by modification by silylation with trimethylchlorosilane (TMCS).

XRD study reveals that the framework of silylated Si-MCM-41 was not destroyed and the long-range order was found to increase upon silylation. The very slight shifting of the main peak (100) to higher $2 \theta^{\circ}$ for calcined and silylated Si-MCM-41 indicates the removal of the organic surfactant template and incorporation of the trimethylsilyl groups, respectively. It also revealed from FTIR spectroscopy and ${ }^{13} \mathrm{C} C P / M A S$ NMR that there is interaction between TMCS and silanol group of wall surface of Si-MCM-41.

\section{References}

Bu, J., \& Rhee, H.-K. (2000). Improvement in Hydrophobicity of Ti-MCM-41 Using a New Silylation Agent MSTFA. Catalysis Letters, 65, 141-145.

Chu, P. P., Reddy, M. J., \& Kao. H.M. (2003). "Novel Composite Polymer Electrolyte Comprising Mesoporous Structured $\mathrm{SiO}_{2}$ and PEO/Li. Solid State Ionics, 156, 141-153.

Corma, A. (1997). From microporous to mesoporous molecular sieve materials and their use in catalysis. Chem. Rev, 97, 2373-2419.

Guo, W., Huang, L., Deng, P., Xue, Z., \& Li, Q. (2001). Characterization of Beta/MCM-41 Composite Molecular Sieve Compared with the Mechanical Mixture. Microporous and Mesoporous Materials, 44-45, 427-434. 
He, N., Yang, C., Dai, Q., Lu, Z., Wang, J., \& Yuan, C. (2000). Studies on the State of Fe and La in MCM-41 Mesoporous Molecular Sieve Materials by TG Analysis and Other Techniques. J. Therm. Anal. Cal, 61, 827-832.

Hoffmann, F., Cornelius, M., Morell, J., \& Froba, M. (2006). Silica-based mesoporous organic-inorganic hybrid materials. Angew. Chem. Int Ed Engl., 45, 3216 - 3251.

Jeong, A.-Y., Koo, S.-M., \& Kim, D.-P. (2000). Characterization of Hydrophobic $\mathrm{SiO}_{2}$ Powders Prepared by Surface Modification on Wet Gel. Journal of Sol-Gel Science and Technology, 19, 483-487.

Juang, L.C., Wang, C.C., \& Lee, C.K. (2006). Adsorption of basic dyes onto MCM-41," Chemosphere, 64, $1920-1928$.

Kang, S.-K., \& Choi, S.-Y. (2000). Synthesis of Low-Density Silica Gel at Ambient Pressure : Effect of Heat Treatment. Journal of Material Science, 35, 4971-4976.

Kleitz, F., Schmidt, W., \& Schuth, F. (2001). Evolution of Mesoporous Materials during The Calcinations Process: Structure and Chemical Behavior. Microporous and Mesoporous Materials. 44-45, 95-105.

Kleitz, F., Schmidt, W., \& Schuth, F. (2003). Calcination Behavior of Different Surfactant-Templated Mesostructured Silica Materials. Microporous and Mesoporous Materials. 65, 1-29.

Kresge, C.T., J.S. Beck, J.S., Vartuli, C., Roth, W.J., Leonowicz, M.E., Scmmitt, K.D., Chu, C.T.W., Olson, D.H., Sheppard, E.W., McCullen, S.B., Higgins, J.B., \& Schlenker, J.L. (1992). A new family of mesoporous molecular sieves prepared with liquid crystal templates. J. Am. Chem. Soc, 114, 10834-10843.

Lazaridis, N.K., Asouhidou, D.D., Triantafyllidis, K.S., \& Matis, K.A. (2009). Adsorption of Remazol Red 3BS from aqueous solutions using APTES- and cyclodextrin-modified HMS-type mesoporous silicas. Colloids and Surfaces A: Physicochem. Eng. Aspects, 346, 83-90.

Lim, M.H., \& Stein, A. (1999). Comparative studies of grafting and direct synthesis of inorganic-organic hybrid mesoporous materials. Chem. Mater, 11, 3285-3295.

Selvaraj, M., Pandurangan, A., Seshadri, K.S., Sinha, P.K., \& Lal, K.B. (2003). Synthesis, Characterization and Catalytic Application of MCM-41 Mesoporous Molecular Sieves containing Zn and Al. Applied Catalysis A: General, 242, 347-364.

Shylesh, S., \& Singh, A.P. (2004). Synthesis, Characterization and Catalytic Activity of Vanadium-Incorporated, -Grafted and -Immobilized Mesoporous MCM-41 in the Oxidation of Aromatics. Journal of Catalysis, 228, 333-346.

Sutra, P., Fajula, F., Brunel, D., Lentz, P., Daelen, G., \& Nagy, J.B. (1999). ${ }^{29} \mathrm{Si}$ and ${ }^{13} \mathrm{C}$ MAS-NMR Characterization of Surface Modification of Micelle-Templated Silicas during the Grafting of Organic Moieties and End-capping. Colloids and Surfaces A: Physicochemical and Engineering Aspects, 158, 21-27.

Zhao, X.S., Lu, G.Q., \& G.J. Millar. (1996). Advances in mesoporous sieve MCM-41. Ind. Eng. Chem. Res, 35, 2075-2090.

Table 1. BET surface areas for as-synthesized, calcined and silylated samples of Si-MCM-41.

\begin{tabular}{|l|c|}
\hline Sample & $\mathbf{S}_{\text {BET }}\left(\mathbf{m}^{\mathbf{2}} \mathbf{~ g}\right)$ \\
\hline As-synthesized Si-MCM-41 & 687.36 \\
\hline Calcined Si-MCM-41 & 1083.55 \\
\hline Silylated Si-MCM-41 & 975.42 \\
\hline
\end{tabular}

The specific BET surface areas of as-synthesized, calcined and silylated Si-MCM-41. The surface area for all the samples were determined by single-point BET method. 


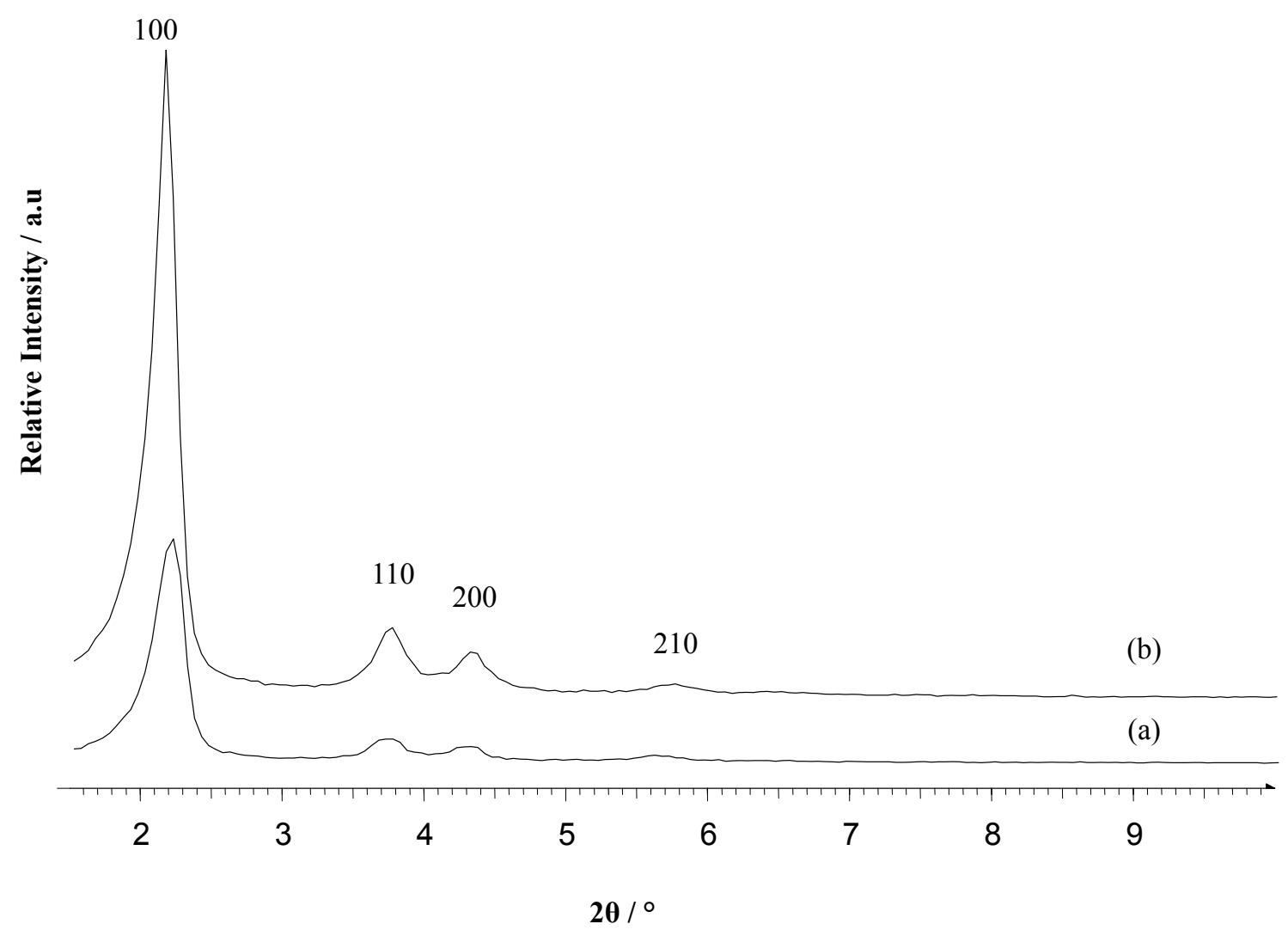

Figure 1. XRD patterns for (a) siliceous MCM-41 and (b) calcined siliceous MCM-41 at $550{ }^{\circ} \mathrm{C}$ in air

The X-ray Diffraction patterns for siliceous MCM-41 before and after calcinations in air at $550{ }^{\circ} \mathrm{C}$. The XRD pattern exhibits four diffraction peaks which could be indexed as (100), (110), (200) and (210) reflections, respectively. This is characteristics of long range ordered hexagonal MCM-41 mesoporous phase. 


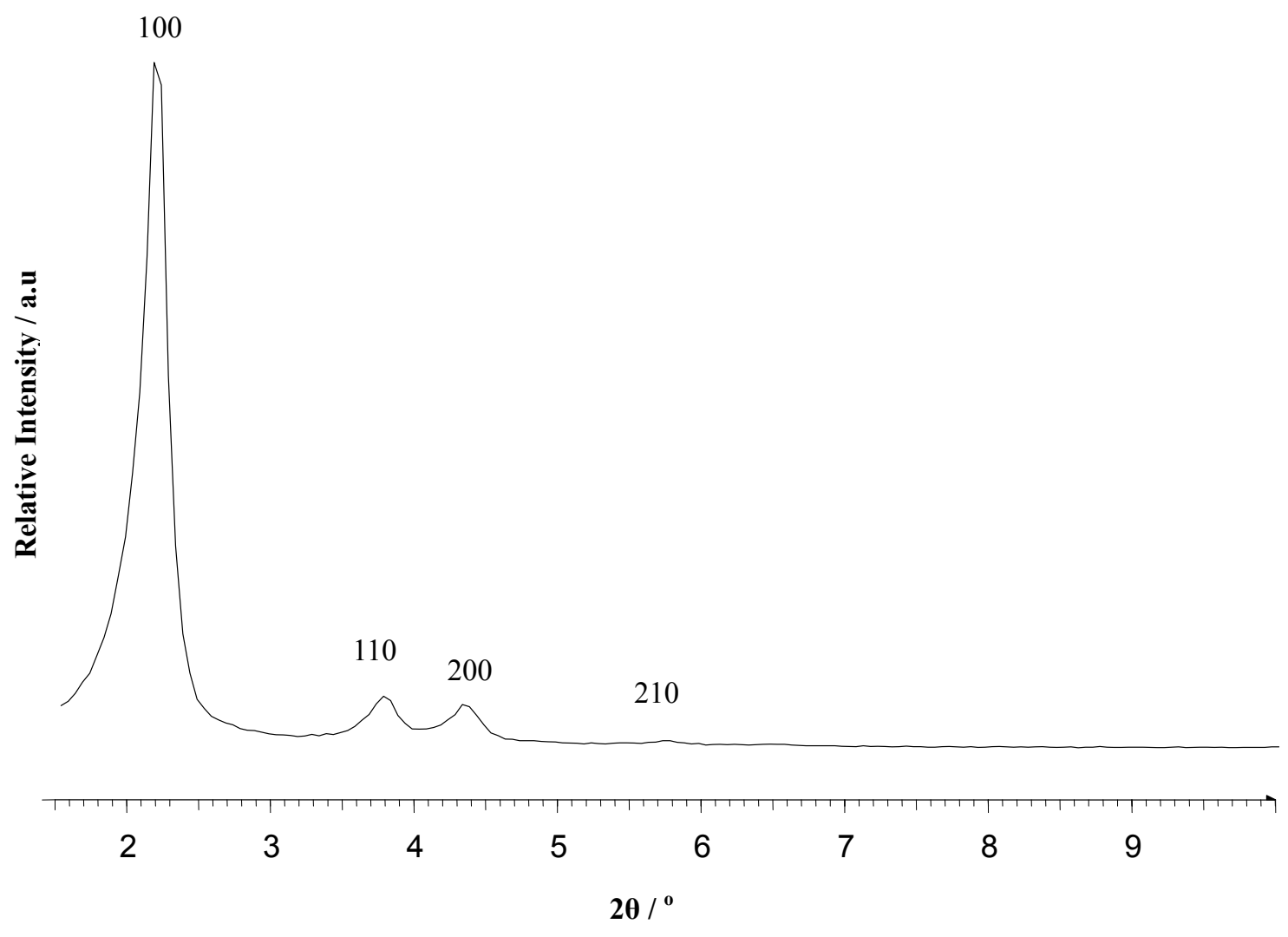

Figure 2. XRD pattern for silylated Si-MCM-41 obtained by modification by silylation with TMCS

The four main peaks still remained even after silylation process with increasing the intensity of (100) peak. XRD patterns suggests that long range order of mesopores is still preserved and the silylation has no adverse effects on the ordered mesoporous structure of MCM-41 and TMCS only interacts on to the surface of calcined siliceous MCM-41. 
(a)

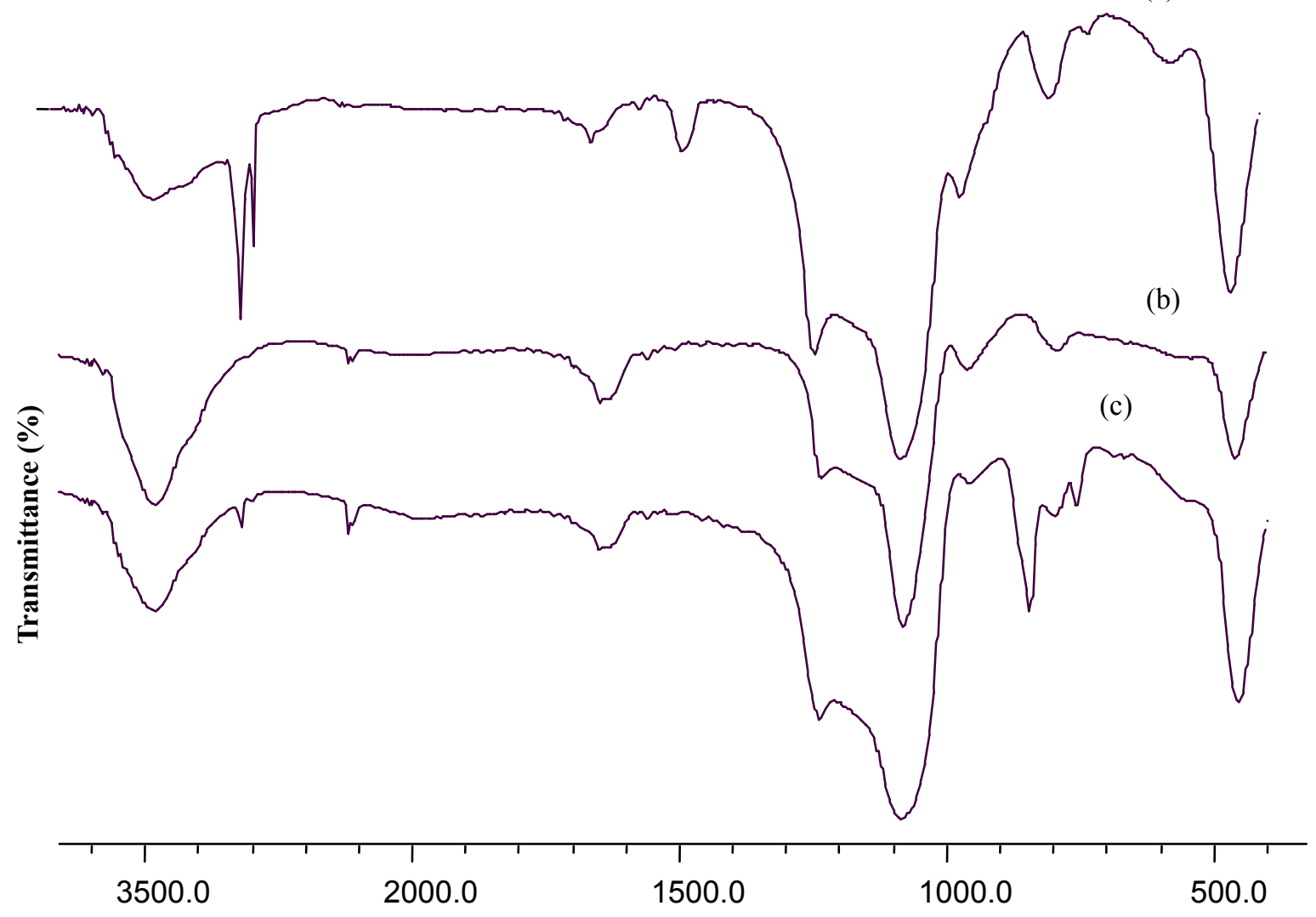

\section{Wavenumber $/ \mathbf{~ c m}^{-1}$}

Figure 3. FTIR spectra for (a) siliceous MCM-41, (b) calcined siliceous MCM-41 and (c) silylated Si-MCM-41

The FTIR spectra of siliceous MCM-41 before and after calcination and the modified product by silylation with trimethylchlorosilane (TMCS) are illustrated in Figure 3. For calcined siliceous MCM-41, the disappearance of peak at $2922.9 \mathrm{~cm}^{-1}$ and $2852.5 \mathrm{~cm}^{-1}$ can be concluded that the calcination at $550{ }^{\circ} \mathrm{C}$ is complete. From the silylated spectrum, the presence of new peaks indicates that there is interaction between TMCS and silanol group on wall surface of MCM-41. 


\section{$-1.111$}

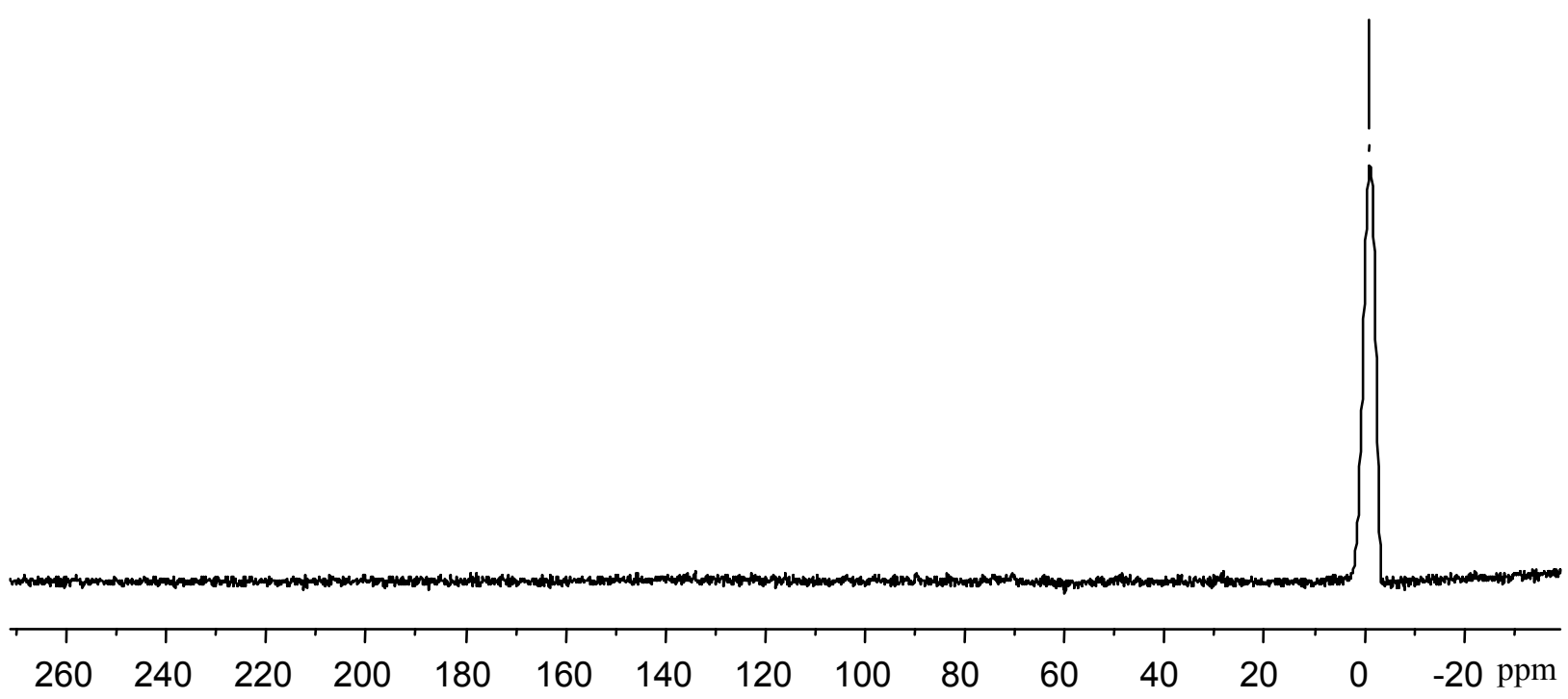

Figue $4 .{ }^{13} \mathrm{C} \mathrm{CP} / \mathrm{MAS}$ NMR spectrum for silylated Si-MCM-41

The existence of one peak corresponds to carbon of methyl group in TMCS that anchored on the silica surface of Si-MCM-41 which indicate that trimethylsilyl groups have been successfully grafted onto the Si-MCM-41.

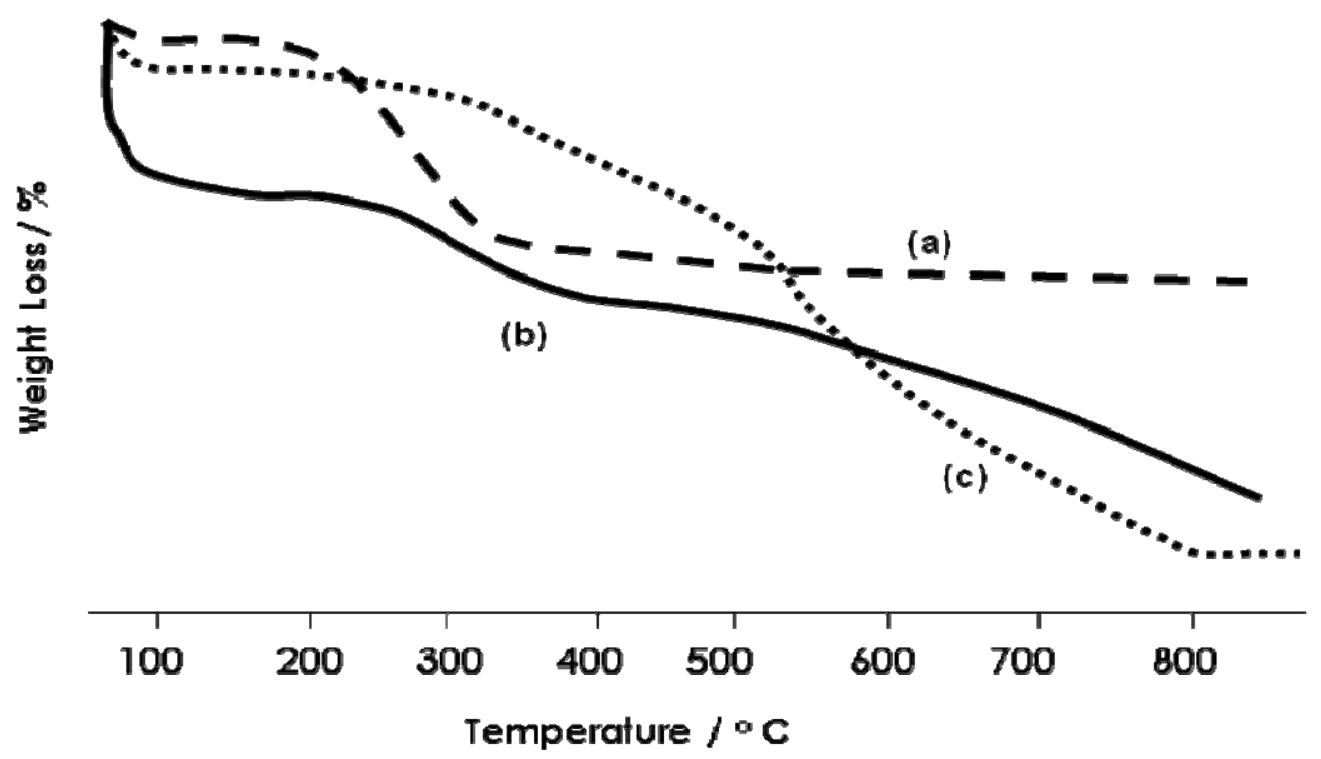

Figure 5. TGA curve of (a) siliceous MCM-41, (b) calcined Si-MCM-41 and (c) silylated Si- MCM-41 in nitrogen atmosphere from $40{ }^{\circ} \mathrm{C}$ to $900{ }^{\circ} \mathrm{C}$ at $20.00{ }^{\circ} \mathrm{C} / \mathrm{min}$

Figure 5 shows the TGA profiles of the (a) siliceous MCM-41, (b) calcined Si-MCM-41 and (c) silylated Si-MCM-41 in nitrogen atmosphere showing three stages of weight loss. The first weight loss due to desorption of physisorbed water on the external surface of the crystallites or occluded in the macropores and mesopores present between the crystallites aggregates. At the temperature range $330-550{ }^{\circ} \mathrm{C}$ is due to residual surfactant decomposition and water loss from the condensation of adjacent silanol group to form siloxane bond. Meanwhile, further decomposition was observed after $550{ }^{\circ} \mathrm{C}$ due to the thermal dehydroxylation of internal surface silanol groups to form siloxane bridges. 